\title{
General Entomology/Intomologia Geral Bioatividade do óleo essencial de Lippia sidoides Cham. (alecrim-pimenta) sobre Callosobruchus maculatus (Fabr.) (Coleoptera: Crysomelidae)
}

\author{
Vanusa Suelma Santos ${ }^{1}{ }^{\bowtie}$, Paulo Henrique Silva $^{2}$ \& Luiz Evaldo Pádua ${ }^{1}$
}

1. Universidade Federal do Piaú. 2. Embrapa Meio-Norte.

\section{EntomoBrasilis 11 (2): 113-117 (2018)}

\begin{abstract}
Resumo. Callosobruchus maculatus (Fabr.), caruncho, é considerado o principal inseto infestante de grãos e sementes de feijão-caupi [Vigna unguiculata (L.) Walp] armazenados por causar danos diretos e indiretos levando perdas econômicas ao agricultor. Derivados botânicos têm sido utilizados na forma de pós, extratos e óleos essenciais no controle de carunchos de grãos armazenados por causa de seu efeito inseticida, repelente, inibidor de alimentação e regulador de crescimento. Neste trabalho avaliou-se a mortalidade, a eficiência e a repelência do óleo essencial de alecrimpimenta (Lippia sidoides Cham.) sobre caruncho do feijão-caupi em diferentes concentrações: 4,0; 3,5; 2,5; 2,0 e o,o $\mu \mathrm{L}$, as quais, no experimento confinado foram pipetadas com uma pipeta automática sobre os grãos de feijão-caupi cv. BRS Guariba e acondicionados em frascos de polietileno com capacidade de $45 \mathrm{~cm}^{3}$ foram adicionados 10 insetos, que ficaram confinados por $96 \mathrm{~h}$. No experimento com chance de escolha, utilizou-se uma arena de seis vias, composta por frascos com a mesma capacidade, quantidade de grãos, insetos e concentrações de óleo: 1,0; 2,0; 3,0 e 4,0 $\mu \mathrm{L}$. Os insetos permaneceram confinados por $24 \mathrm{~h}$. O delineamento utilizado para os dois experimentos foi inteiramente casualizado com cinco repetições. Os dados foram submetidos à análise variância (ANOVA) e as médias comparadas pelo teste Tukey a $5 \%$ de probabilidade através do programa ASSISTAT VERSÃO 7.5. A eficiência foi calculada através da fórmula de Abbott. Todas as concentrações apresentaram ação inseticida, foram repelentes e eficientes no controle de C. maculatus.
\end{abstract}

Palavras-chave: Alecrim pimenta; caruncho; Inseticidas botânicos; repelência; Vigna unguiculata.

\section{Bioactivity of the essential oil of Lippia sidoides Cham. (rosemary-pepper) on the Callosobruchus maculatus (Fabr.) (Coleoptera: Chysomelidae)}

\begin{abstract}
Callosobruchus maculatus (Fabr.), weevil, is considered the main insect infesting of grains and seeds of cowpea-been [Vigna unguiculata (L.) Walp] stored for causing direct and indirect damages leading to economic losses to the producer. Botanical derivatives have been used in the form of powders, extracts and essential oils in the control of stored grain weevil because of their insecticidal effect, repellent, feed inhibitor and growth regulator. In this work, mortality, efficiency and repellency of rosemary essential oil (Lippia sidoides Cham.) on cowpea-been weevil were evaluated in different concentrations: 4.0; $3.5 ; 2.5 ; 2.0$ and $0.0 \mu \mathrm{L}$, which, in the confined experiment, were pipetted with an automatic pipette on the grains of cowpea-beans cv. BRS Guariba in polyethylene flasks with a capacity of $45 \mathrm{~cm}^{3}$ and later, 10 insects were added, which were confined for 96 h. In the experiment with chance of choice, a six-way arena was used, consisting of bottles with the same capacity, quantity of grains, insects and oil concentrations: 1,$0 ; 2,0 ; 3,0$ and 4,0 $\mu \mathrm{L}$. Insects remained confined for $24 \mathrm{~h}$. The design used for the two experiments was completely randomized with five replicates. The data were submitted to analysis of variance (ANOVA) and the averages were compared by the Tukey test at $5 \%$ of probability through the ASSISTAT VERSION 7.5 program. Efficiency was calculated using the Abbott formula. All the concentrations presented insecticide action, were repellent and efficient in the control of $C$. maculatus.
\end{abstract}

Keywords: Botanical insecticides; repellency; Rosemary; Vigna unguiculata; Weevil.

(0) feijão-caupi Vigna unguiculata (L.) Walp. é uma leguminosa de grande importância para o desenvolvimento agrícola de muitas regiões tropicais e subtropicais no mundo. No Brasil, as regiões Norte e Nordeste são as principais produtoras dessa leguminosa (FROTA et al. 2008), sendo cultivada por pequenos produtores como fonte de renda ou sustentabilidade, por ser fonte de proteína, vitaminas e sais minerais (CRUZ et al. 2012).

Apesar da ampla adaptabilidade às condições de solo e clima, os problemas fitossanitários presentes na cultura do feijão-caupi são freqüentes e afetam significativamente a produção (GALLO et al. 2002) e o armazenamento pelos danos causados por Callosobruchus maculatus (Fabr.) (Coleoptera: Chysomelidae: Bruchinae) considerado o principal inseto que ataca os grãos e sementes de feijão-caupi (Marsaro Junior \& Vilarinho 2011).

$\mathrm{O}$ ataque desse inseto pode ocorrer no campo quando as fêmeas ovipositam sobre os grãos de vagens deiscentes, ou quando as fêmeas voam até os depósitos de armazenamento presentes nas propriedades rurais (MeLo et al. 2014), diminuindo o valor nutricional, a qualidade e germinação das sementes (AzEvEDo et

\section{Edited by:}

Anderson Gonçalves Silva

\section{Article History:}

Received: 29.vii.2017

Accepted: $14 . v .2018$
Corresponding author:

Vanusa Suelma Santos

vanfla1@hotmail.com

( ) No ORCID record
Funding agencies:

$\leftrightarrow$ Without funding declared 
al. 2007), decorrentes da colonização no interior dos grãos pelas larvas do inseto (Almeida et al. 2005).

Não existem produtos registrados no Ministério da Agricultura Pecuária e Abastecimento (MAPA) para o controle de $C$. maculatus, na cultura do feijão- caupi, mas é comum o uso de inseticidas fumigantes (MARSARO JUNIOR \& VILARINHO 2011), OS quais quando utilizados de forma intensiva podem provocar resistência de insetos ao princípio ativo, contaminação dos grãos por acúmulo de resíduos tóxicos e contaminação do meio ambiente (Pimentel et al. 2010).

Como alternativa aos inseticidas sintéticos, métodos alternativos a partir de recursos naturais oriundos de partes de plantas estão sendo utilizados por agricultores familiares por apresentarem ação inseticida, repelência, inibição na alimentação, reguladores de crescimento (SILva et al. 2011), como alternativa de baixo custo e ecologicamente adequada ao controle de insetos em grãos armazenados pelo seu potencial bioativo (IsMAN 2006), apresentarem baixa toxicidade para o homem e animais, fáceis de serem adquiridos e utilizados (PEREIRA et al. 2008).

Neste contexto, a bioatividade do gênero Lippia já foi comprovada em alguns estudos e indicam a potencialidade dos seus constituintes químicos sobre Aedes aegypti (CARvalHo 2003) e insetos em grãos armazenados (Olivero-Verbel et al. 2009; LIMA et al. 2011; CASTRO 2013).

Desta forma, considerando a necessidade de se obter novos resultados por meio de produtos vegetais como recurso alternativo no controle de C. maculatus em vista aos prejuízos econômicos que ele causa, este trabalho teve por objetivo avaliar a mortalidade, a eficiência e a repelência do óleo essencial de alecrim pimenta (Lippia sidoides Cham.) sobre C. maculatus em feijão-caupi armazenado.

\section{MATERIAL E MÉTODOS}

Ensaios com e sem chance de escolha foram realizados de 06 de julho de 2015 a 26 de fevereiro de 2016 no laboratório de entomologia da Embrapa Meio-Norte, em Teresina-PI ( $05^{\circ}$ 05'S; $42^{\circ} 29^{\prime} \mathrm{W}$ e $72 \mathrm{~m}$ de altitude), sob condições controladas em estufa incubadora, com temperatura de $27 \pm 2{ }^{\circ} \mathrm{C}$, umidade relativa $60 \pm 10 \%$ e fotofase de $12 \mathrm{~h}$.

Para a criação dos insetos e montagem dos experimentos foi utilizado grãos da cultivar de feijão-caupi BRS Guariba. Para impedir possíveis infestações oriundas do campo, os grãos foram acondicionados em sacos plásticos com capacidade de $2 \mathrm{~kg}$ e mantidos em freezer sob temperatura de $-10{ }^{\circ} \mathrm{C}$ por 30 dias. Em seguida, $300 \mathrm{~g}$ de grãos foram transferidos para frascos de polietileno com capacidade para $1 \mathrm{~kg}$, fechados com tampa plástica, na qual foi feita uma abertura central, adaptando-se um tecido "voil" para permitir a aeração interna e posteriormente, esses frascos ficaram acondicionados em temperatura ambiente por dois dias para atingirem o equilíbrio higroscópico.

Após esse período, os insetos foram confinados durante cinco dias para realizarem a postura, em seguida, retirados e os frascos mantidos em estufa incubadora até a emergência da geração f1, a qual foi utilizada para novas infestações. Este procedimento foi realizado para garantir a quantidade de insetos necessários nos experimentos.

Para obtenção do óleo essencial, as folhas de alecrim-pimeta ( $L$. sidoides) foram coletadas no campo experimental da Embrapa Meio-Norte, após levadas ao laboratório foram cortadas, pesadas e submetidas ao processo de hidrodestilação por meio do aparelho de Clevenger por um período de $3 \mathrm{~h}$. O óleo essencial foi coletado por meio de uma pipeta de Pasteur e acondicionado em recipiente de vidro âmbar, vedado e armazenado em freezer à temperatura de $-10{ }^{\circ} \mathrm{C}$ até ser utilizado nos bioensaios.
No experimento sem chance de escolha onde se avaliou o potencial do óleo de folhas de $L$. sidoides no controle de $C$. maculatus, o delineamento experimental foi o inteiramente casualizado com cinco repetições e cinco tratamentos representados pelas concentrações, 4,0; 3,5; 3,0; 2,5; 2,0 $\mu \mathrm{L}$ de óleo de $L$. sidoides e uma testemunha. As parcelas consistiram em frasco de polietileno com capacidade de $45 \mathrm{~cm}^{3}$, contendo as concentrações do óleo, mais $10 \mathrm{~g}$ de grãos de feijão-caupi cv BRS Guariba e dez insetos de C. maculatus com idade entre o à $48 \mathrm{~h}$, os quais permaneceram confinados por $96 \mathrm{~h}$. Decorrido esse período, os insetos foram contabilizados por tratamento em vivos e mortos e desprezados. Após quinze dias da infestação inicial foram feitas as leituras dos ovos viáveis e inviáveis. Transcorrido 25 dias da infestação, as observações foram diárias no sentido de quantificar e desprezar os insetos emergidos. Essa contagem findou quando por cinco dias consecutivos não havia mais emergência de insetos.

No experimento com chance de escolha onde se avaliou o potencial de repelência do óleo de folhas de $L$. sidoides sobre C. maculatus, o delineamento experimental foi o inteiramente casualizado com cinco tratamentos e cinco repetições. Na montagem do experimento foram utilizadas cinco arenas, cada uma correspondeu a uma repetição do experimento contendo seis frascos circulares de polietileno com capacidade aproximada de $45 \mathrm{~cm}^{3}$. O frasco central da arena era interligado aos cinco periféricos por meio de tubos plásticos de 1,0 cm de diâmetro e $10 \mathrm{~cm}$ de comprimento. Em cada frasco periférico foi adicionado $10 \mathrm{~g}$ de grãos de feijão-caupi cv BRS Guariba, após adicionou-se as concentrações de óleo: 1,0; 2,0; 3,0 e 4,0 $\mu \mathrm{L}$ e misturadas por meio de um bastão de vidro para uniformização. No recipiente central de cada arena foram liberados 50 insetos não sexados de C. maculatus com idade entre 0 à $48 \mathrm{~h}$.

Depois de $24 \mathrm{~h}$ da liberação dos insetos foi quantificado o número de insetos atraídos por tratamentos e em seguida, desprezados. Avaliou-se o número de insetos atraídos e índice de repelência (IR), onde: $\mathrm{IR}=2 \mathrm{G} /(\mathrm{G}+\mathrm{P})$, onde $\mathrm{IR}=$ índice de repelência, $\mathrm{G}=\%$ de insetos atraídos por tratamento e $\mathrm{P}=\%$ de insetos atraídos na testemunha. Os valores de IR variam entre zero e dois, indicando: $\mathrm{IR}=1, \mathrm{O}$, neutra; $\mathrm{IR}>1, \mathrm{O}$, atraente $\mathrm{e} \mathrm{IR}<1, \mathrm{O}$, repelente.

Como margem de segurança para classificação, o desvio padrão (DP) de cada tratamento foi adicionado ou subtraído do valor 1,0 (indicativo de neutralidade). Desta forma, cada tratamento só foi considerado repelente ou atraente quando o IR estava fora do intervalo 1,0 \pm DP (MAZzonETo \& VeNDRAMIM 2003).

Aos 15 dias da infestação inicial, o número de ovos viáveis (coloração branca opaca) e inviáveis (coloração hialina) presentes nos grãos foi avaliado com auxílio de microscópio estereoscópico e aos 25 dias, as observações passaram a ser diárias, cujo objetivo foi quantificar e desprezar os insetos emergidos. Os dados de mortalidade, total de ovos, ovos inviáveis e emergência de insetos foram submetidos à análise de variância pelo teste $\mathrm{F} \mathrm{e}$ as médias comparadas pelo teste Tukey a $5 \%$ de probabilidade. Os dados de mortalidade também foram submetidos à análise de regressão através do programa Microsoft Excel ${ }^{\mathrm{TM}}$. A eficiência dos tratamentos foi calculada por meio da fórmula de Аввотт (1925).

\section{RESULTADOS E DISCUSSÃO}

Verificou-se que os maiores percentuais de mortalidade foram observados nas concentrações de 4,0; 3,5 e 3,0 $\mu \mathrm{L} / 45 \mathrm{~cm}^{3}$ que variou de 98 a 84\%, respectivamente sem diferença significativa entre elas (Tabela 1).

A eficiência foi estatisticamente igual em todas as concentrações variando de 98 a 65\%. O total de ovos depositados foi inversamente proporcional à concentração, variando de 2,6 a 282,6 ovos, respectivamente para a maior concentração $\left(4,0 \mu \mathrm{L} / 45 \mathrm{~cm}^{3}\right)$ e testemunha. A inviabilidade dos ovos foi 
Tabela 1. Percentual de mortalidade, eficiência, média de total de ovos, percentual de ovos inviáveis e média de adultos emergidos de Callosobruchus maculatus em grãos de feijão-caupi cv BRS Guariba tratados com diferentes concentrações de óleo essencial de folhas de Lippia sidoides em teste sem chance de escolha.

\begin{tabular}{|c|c|c|c|c|c|}
\hline $\begin{array}{c}\text { Concentrações de } \\
\text { óleo }\end{array}$ & Mortalidade $^{1}(\%)$ & Eficiência $^{1}$ (\%) & Total de ovos ${ }^{1}$ & Ovos inviáveis ${ }^{1}(\%)$ & $\begin{array}{c}\text { Adultos } \\
\text { Emergidos }^{1}\end{array}$ \\
\hline $4,0 \mu \mathrm{L}$ & 98,0 a & $98 \mathrm{a}$ & $2,6 \mathrm{c}$ & $100,0 \mathrm{a}$ & $0,0 \mathrm{~b}$ \\
\hline $3,5 \mu \mathrm{L}$ & $90,0 \mathrm{ab}$ & $90 \mathrm{a}$ & $9,6 \mathrm{~b} \mathrm{c}$ & $100,0 \mathrm{a}$ & $0,0 \mathrm{~b}$ \\
\hline $3,0 \mu \mathrm{L}$ & $84,0 \mathrm{ab}$ & $83 a$ & $11, \mathrm{o} \mathrm{b} \mathrm{c}$ & $100,0 \mathrm{a}$ & $0,0 \mathrm{~b}$ \\
\hline $2,5 \mu \mathrm{L}$ & $62, \mathrm{o} \mathrm{b}$ & $61 \mathrm{a}$ & $20,4 \mathrm{~b}$ & 100,0 a & $0,0 \mathrm{~b}$ \\
\hline $2,0 \mu \mathrm{L}$ & $66,0 \mathrm{~b}$ & $65 \mathrm{a}$ & $20,6 \mathrm{~b}$ & $20,0 \mathrm{~b}$ & $0,0 \mathrm{~b}$ \\
\hline Testemunha & $2, \mathrm{O} \mathrm{c}$ & - & 282,6 a & $7,9 \mathrm{~b}$ & 239,8 a \\
\hline $\mathbf{C V}$ & 25,9 & 13,7 & $\mathbf{2 4 , 9}$ & 24,6 & 27,6 \\
\hline
\end{tabular}

${ }^{1}$ Médias seguidas de mesma letra na coluna não diferem estatisticamente entre si pelo teste Tukeya $5 \%$ de probabilidade. Dados transformados em $\sqrt{ } \mathrm{x}$.

de $100 \%$ entre as concentrações de 4,o $\mu \mathrm{L} / 45 \mathrm{~cm}^{3}$ e $2,5 \mu \mathrm{L} / 45$ $\mathrm{cm}^{3}$, entretanto mesmo na concentração de $2,0 \mu \mathrm{L} / 45 \mathrm{~cm}^{3}$, que apresentou inviabilidade de $20 \%$ dos ovos, não permitiu, assim como nas demais concentrações, emergência de adultos indicando possivelmente, uma ação ovicida do óleo estudado (Tabela 1).

CAStro (2013) avaliou a ação inseticida de óleos essenciais da planta fresca e desidratada de $L$. sidoides na concentração de $20 \mu \mathrm{L} / \mathrm{L}$ de ar e constatou que eles provocaram mortalidade de $69,17 \%$ e $73,33 \%$ e redução na postura com um total de 80,17 e 72,33 ovos, respectivamente, porém sem emergência de adultos. Estes resultados assemelham-se aos encontrados nesse trabalho, pois em qualquer uma das concentrações estudadas, embora não tenha causado $100 \%$ de mortalidade dos insetos, não permitiu emergência de C. maculatus. Para Silva et al. (2012), os compostos secundários podem agir de diferentes formas sobre os insetos tais como repelentes, inibidores de alimentação, oviposição, perturbadores do crescimento, desenvolvimento e reprodução.

Apesar dos relevantes resultados encontrados neste trabalho, existem poucas pesquisas sobre a espécie $L$. sidoides no controle de $C$. maculatus. No entanto, diversas investigações têm comprovado a eficácia de espécies do gênero Lippia sobre C. maculatus e outros insetos de grãos armazenados (PEREIRA et al. 2008), os quais constataram que o óleo essencial de Lippia gracilis Schauer causou mortalidade de $100 \%$ de C. maculatus e inviabilizou a emergência de insetos adultos em todas as concentrações estudadas1o, 20, 3040 e $50 \mu \mathrm{L} / 120 \mathrm{~cm}^{3}$.

Resultados semelhantes também foram encontrados em outro trabalho realizado por PEREIRA et al. (2009) onde avaliaram a influência do período de armazenamento do caupi tratados com óleos essenciais e fixos no controle de $C$. maculatus e concluíram que na concentração de $50 \mu \mathrm{L} / 120 \mathrm{~cm}^{3}$, após impregnação dos grãos, causou mortalidade de $100 \%$ do referido inseto não permitindo emergência dos mesmos, bem como o óleo essencial de Lippia multiflora nas Moldenke doses de $10 \mu \mathrm{L} / \mathrm{L}$ e $20 \mu \mathrm{L} / \mathrm{L}$ proporcionou mortalidade de $80 \%$ e $100 \%$, respectivamente de C. maculatus (Ilboudo et al. 2010). Estes resultados corroboram com os encontrados por este trabalho.

Mahmoudvand et al. (2011) usaram óleo essencial de Lippia citriodora Kunth na forma fumigação sobre adultos de $C$. maculatus e concluíram que nas concentrações de 114,24; 142,$8 ; 171,36 ; 257,05$ e $285,8 \mu \mathrm{L} / \mathrm{L}$ ar e tempo $3,6,9,12$ e $24 \mathrm{~h}$, respectivamente, provocou $85 \%$ de mortalidade de insetos em 24 $\mathrm{h}$ na concentração de $285,8 \mu \mathrm{L} / \mathrm{L}$. Estes resultados evidenciam o espectro de ação inseticida que o gênero Lippia apresenta sobre o inseto estudado.
Em trabalho realizado por Riveraet al (2004) com Acanthocelides obtectus Say utilizando o óleo essencial de Lippia alba Miller nas concentrações de 2 e $4 \mathrm{~mL} / 200 \mathrm{~cm}^{3}$ verificaram que aos 30 e 60 dias houve mortalidade de $100 \%$ dos insetos.

Observa-se que embora sejam espécies distintas do mesmo gênero, sobre $C$. maculatus e outros insetos de grãos armazenados, apresentaram resultados semelhantes aos encontrados neste trabalho. Portanto, a redução na emergência de adultos nos grãos tratados com óleo essencial de $L$. sidoides pode estar associada à possível ação inseticida desse óleo sobre a fase de ovo/ou larva de C. maculatus. Segundo Guimarães et al. (2014), o efeito do óleo essencial de L. sidoides deve-se à presença do carvacrol em sua constituição. Para CREDLAND (1992) a oclusão da micrópila por alguns óleos pode explicar o efeito ovicida/larvicida, bem como a susceptibilidade do ovo de Callosobruchus aos óleos em relação a outros bruquídeos que não possuem essa estrutura.

As substâncias responsáveis pela diferença na toxicidade sobre os insetos citados estão relacionadas com a constituição química das espécies e sua ação sobre a biologia dos mesmos. Por outro lado, cada espécie de planta tem uma variação no volume de seus constituintes químicos em função de diversos fatores ambientais como menciona (SOARES \& TAVARES-DiAS 2013).

A representação gráfica da regressão aponta efeito linear crescente de mortalidade com o aumento das concentrações do óleo essencial de folhas de L. sidoides. O coeficiente angular (b) da equação mostra que, com a adição de 1,o $\mu \mathrm{L}$ de óleo, há um aumento de 24,1\% na mortalidade de C. maculatus (Figura 1).

Com relação à repelência, o número médio de insetos atraídos foi menor nas concentrações de 4,o; 3,0 e 2,o $\mu \mathrm{L} / 45 \mathrm{~cm}^{3}$ do óleo essencial de folhas de $L$. sidoides. Nessas mesmas concentrações, o número total de ovos foi menor que os demais tratamentos e não permitiram a emergência de insetos. A menor concentração 1,0 $\mu \mathrm{L} / 45 \mathrm{~cm}^{3}$, embora tenha apresentado um número significativo de ovos, não permitiu emergência de adultos, o que mostra possivelmente, uma ação ovicida/larvicida do óleo. Todas as concentrações apresentaram índice de repelência menor que 1,0, sendo classificadas como repelentes (Tabela 2).

A ação repelente é muito importante no manejo de insetos de grãos armazenados com óleos essenciais, pois quanto maior a repelência, menor infestação dos grãos, menor o número de ovos e de insetos emergidos, o que foi observado nesse estudo.

CASTRo (2013) observou resultado contrário utilizando o óleo de $L$. sidoides. A autora concluiu que a referida espécie, embora tenha apresentado o menor número de insetos atraídos, apresentouse como neutra a $C$. maculatus com uso da concentração de 20 $\mu \mathrm{L} / \mathrm{L}$ de ar. Esta diferença nos resultados pode ter ocorrido em 


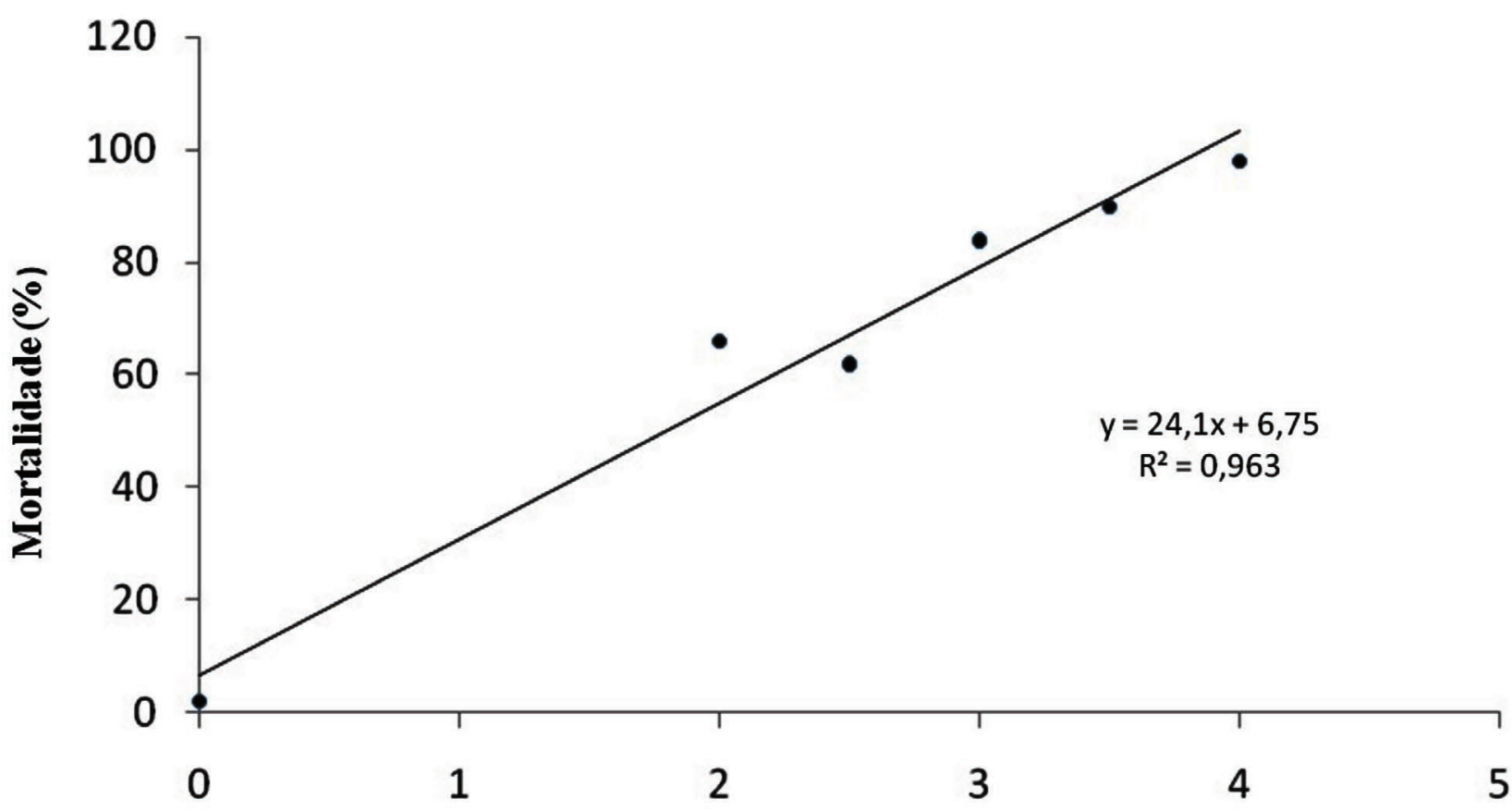

\section{Concentrações $(\mu \mathrm{L})$}

Figura 1. Representação gráfica e equação de regressão entre concentrações de óleo de folhas Lippia sidoides e percentual de mortalidade de Callosobruchus maculatus.

Tabela 2. Número médio de Callosobruchus maculatus atraídos, índice de repelência, classificação, número total de ovos, ovos inviáveis e insetos emergidos em grãos de feijão- caupi cv BRS Guariba tratados com diferentes concentrações de óleo de folhas de Lippia sidoides em teste com chance de escolha.

\begin{tabular}{|c|c|c|c|c|c|c|}
\hline $\begin{array}{c}\text { Concentrações } \\
\text { de óleo }\end{array}$ & $\begin{array}{l}\text { Insetos } \\
\text { atraídos }^{1}\end{array}$ & $\begin{array}{c}\text { Índice de } \\
\text { Repelência } \\
\quad( \pm \mathrm{DP})\end{array}$ & Classificação & Total de ovos ${ }^{1}$ & Ovos inviáveis $^{1}$ & $\begin{array}{c}\text { Insetos } \\
\text { emergidos }^{1}\end{array}$ \\
\hline $4,0 \mu \mathrm{L}$ & $0,8 \mathrm{c}$ & $0,06 \pm 0,03$ & Repelente & $0,2 \mathrm{c}$ & $0,2 \mathrm{c}$ & $\mathrm{o}, \mathrm{o} \mathrm{b}$ \\
\hline $3,0 \mu \mathrm{L}$ & $2,4 \mathrm{c}$ & $0,17 \pm 0,07$ & Repelente & $0,4 \mathrm{c}$ & $0,4 \mathrm{c}$ & $0,0 \mathrm{~b}$ \\
\hline $2,0 \mu \mathrm{L}$ & $2,8 \mathrm{c}$ & $0,20 \pm 0,16$ & Repelente & $1,4 \mathrm{c}$ & $1,4 \mathrm{bc}$ & $\mathrm{o}, \mathrm{o} \mathrm{b}$ \\
\hline $1,0 \mu \mathrm{L}$ & $11,4 \mathrm{~b}$ & $0,58 \pm 0,14$ & Repelente & $59,6 \mathrm{~b}$ & $10,4 \mathrm{~b}$ & $\mathrm{o}, \mathrm{o} \mathrm{b}$ \\
\hline Testemunha & 26,6 a & $1,00 \pm 0,00$ & Neutra & 431,0 a & $48,0 a$ & $241,8 \mathrm{a}$ \\
\hline CV & 24,2 & & & 36,3 & 34,2 & $\mathbf{3 3 , 0}$ \\
\hline
\end{tabular}

${ }^{1}$ Médias seguidas de mesma letra na coluna não diferem estatisticamente entre si pelo teste Tukey a $5 \%$ de probabilidade. $\mathrm{DP}=$ desvio padrão. Dados transformados em $\sqrt{ } \mathrm{x}$.

virtude da coleta de folhas em idades diferentes da planta, fato que pode interferir na quantidade dos constituintes químicos da mesma (SOARES \& TAVARES-DiAS 2013).

Resultados semelhantes ao deste trabalho foi observado por Olivero-Verbel et al. (2009) que avaliaram a atividade repelente do óleo essencial de Lippia origanoides Kunth. aplicadas sobre papel filtro e verificaram que o mesmo apresentou atividade repelente significativa com $94 \%$ e $98,0 \%$, respectivamente, sobre Tribolium castaneum (Herbst) na concentração de $0,2 \mu \mathrm{L} / \mathrm{cm}^{2}$.

Analisando os parâmetros avaliados, pode-se concluir que o óleo essencial de $L$. sidoides é bioativo no manejo de $C$. maculatus com ação inseticida e repelente.

\section{REFERÊNCIAS}

Abbott, W.S., 1925. A method of computing the effectiveness of an insecticide. Journal Economic Entomology. DOI: https://doi.org/10.1093/jee/18.2.265a.

Almeida, F.A.C., S.A. Almeida, N.R. Santos, J.P. Gomes \& M.E.R. Araujo, 2005. Efeito de extratos alcoólicos de plantas sobre o caruncho do feijão Vigna(Callosobruchus maculatus). Revista
Brasileira de Engenharia Agrícola e Ambiental, 9: 585-590. DOI: https://doi.org/10.1590/s1415-43662005000400023.

Azevedo, F.R., A.C.L. Leitão \& M.A.A. Lima, 2007. Eficiência de produtos naturais no controle de Callosobruchus maculatus em feijão-caupi (Vigna unguiculata (L.) Walp.) armazenado. Revista Ciências Agronômica, 38: 182-187.

Carvalho, A.F.U., V.M.M. Melo, A.A. Craveiro, M.I.L. Machado, M.B. Bantim \& E.F. Rabelo, 2003. Larvicidal activity of the essential oil from Lippia sidoides Cham. Against Aedes aegypti Linn. Memórias do Instituto Osvaldo Cruz, 98: 569-571. DOI: https://doi.org/10.1590/s0074-02762003000400027.

Castro, M.J.P. de, 2013. Efeito de genótipo de feijão-caupi e de espécies botânicas em diferentes formulações sobre Callosobruchus maculatus (Fabr.). Tese (Doutorado em Agronomia - Proteção de Plantas). Universidade Estadual Paulista, Faculdade de Ciências Agronômica. $131 \mathrm{f}$.

Credland, P.F., 1992. The structure of Bruchid eggs may explain the ovicidal effect of oils. Journal of Stored Products Research, 28: 01-09. DOI: https://doi.org/10.1016/0022474X(92)90025-1.

Cruz, C.S.A., E.RL. Pereira, L.M.M. Lima, M.B. Medeiros \& J.P. Gomes, 2012. Repelência do Callosobruchus maculatus (Coleoptera: Bruchidae) sobre grãos de feijão-caupi tratados 
com óleos vegetais. Revista Verde de Agroecologia e Desenvolvimento Sustentável, 7: 01-05.

Frota, K.M.G., R.A.M. Soares \& J.A.G. Arêas, 2008. Composição química do feijão caupi (Vigna unguiculata L.) Walp.) cultivar BRS- Milênio. Ciência e Tecnologia de Alimento, 28: 470-476. DOI: https://doi.org/10.1590/s0101-20612008000200031.

Gallo, D., O. Nakano, S. Silveira-Neto, R.P.L. Carvalho, G.C. Batista, E. Berti Filho, J.R.P. Parra, R.A. Zucchi, S.B. Alves, J.D. Vendramim, L.C. Marchini, J.R.S. Lopes \& C. Omoto, 2002. Entomologia agrícola. Piracicaba: FEALQ, 920 p.

Guimarães, L.G.L., M.G. Cardoso, R.M. Souza, A.B. Zacaroni \& G.R. Santos, 2014. Óleo essencial de Lippia sidoides nativas de Minas Gerais: Composição, estruturas secretoras e atividade antibacteriana, Revista Ciência Agronômica, 45: 267-275. DOI: https://doi.org/10.1590/s1806-66902014000200006.

Ilboudo, Z., L.C.B. Dabiré, R.C.H. Nebié, I.O. Dicko, S. Dugravot, A.M. Cortesero \& A. Sanon, 2010. Biological activity and persistence of four essential oils towards the main pest of stored cowpeas, Callosobruchus maculatus (F.) (Coleoptera: Bruchidae). Journal of Stored Products Research, 46: 124128. DOI: https://doi.org/10.1016/j.jspr.2009.12.002.

Isman, M.B., 2006. Botanical insecticides, deterrents, and repellents in modern agriculture and increasingly regulated world. Annual Review of Entomology, 51: 45-66. DOI: https://doi.org/10.1146/annurev.ento.51.110104.151146.

Lima, R.K., M.G. Cardoso, J.C. Moraes, S.M. Carvalho, V.G. Rodrigues \& L.G.L. Guimarães, 2011. Chemical composition and fumigant effect of essential oil of Lippia sidoides Cham. and monoterpenes against Tenebrio molitor (L.) (Coleoptera: Tenebrionidae). Ciência e Agrotecnologia, 35: 664-671. DOI: https://doi.org/10.1590/s1413-70542011000400004.

Mahmoudvand, M., H. Abbasipour, M. H. Hosseinpour, F. Rastegar \& M. Basij, 2011. Using some plant essential oils as natural fumigants against adults of Callosobruchus maculatus (F.) (Coleoptera: Bruchidae). Munis Entomology \& Zoology, 6: 150-154.

Marsaro Junior, A.L. \& A.A. Vilarinho, 2011. Resistência de cultivares de feijão-caupi ao ataque de Callosobruchus maculatus (Coleoptera: Crysomelidae: Bruchinae) em condições de armazenamento. Revista Acadêmica Ciência Animal,9:51-55.DOI:https://doi.org/10.7213/cienciaanimal. v9i1.11078.

Mazzonetto, F. \& J.D. Vendramim. 2003. Efeito de pós de origem vegetal sobre Acanthoscelides obtectus (Say) (Coleoptera: Bruchidae) em feijão armazenado. Neotropical Entomology, 32: 145-149. DOI: https://doi.org/10.159o/ s1519-566x2003000100022.
Melo, B.A., A.J. Molina-Rugama, D.T. Leite, M.S.Godoy \& E.L. Araujo, 2014. Bioatividade de pós de espécies vegetais sobre a reprodução de Calllosobruchus maculatus (Coleoptera: Bruchidae). Bioscience Journal, 30: 346-353.

Olivero-Verbel, J., K. Caballero-Gallardo, B. JaramilloColorado \& E.E. Stashenko, 2009. Actividad repelente de los aceites essenciales de Lippia origanoides, Citrus sinenses y Cymbopogon nardus cultivadas em Colombia frente a Tribolium castaneum Herbst. Revista de La Universidad Industrial de Santander, 41: 244-250.

Pereira, A.C.R.L., J.V. Oliveira, M.G.C. Gondim Junior \& C.A.G. Câmara, 2008. Atividade inseticida de óleos essenciais e fixos sobre Callosobruchus maculatus (Coleoptera: Bruchidae) em grãos de caupi [Vigna unguiculata (L.) Walp.]. Ciência e Agrotecnologia, 32: 717-724. DOI: https://doi.org/10.1590/ s1413-70542008000300003.

Pereira. A.C.R.L., J.V. Oliveira, M.G.C. Gondim-Junior \& C.AG. Câmara, 2009. Influência do período de armazenamento do caupi [Vigna unguiculata (L.) Walp.], tratado com óleos essenciais e fixos, no controle de Callosobruchus maculatus (Fabricius, 1775) (Coleoptera: Chrysomelidae: Bruchinae). Ciência e Agrotecnologia, 33: 319-325. DOI: https://doi.org/10.1590/s1413-70542009000100044.

Pimentel, M.A.G., L.R.D.A. Faroni, F.H. Silva, M.D. Batista \& R.N.C. Guedes, 2010. Spread of phosphine resistance among brazilian populations of three species of stored products insects. Neotropical Entomology, 39: 101-107. DOI: https://doi.org/10.1590/s1519-566x2010000100014.

Rivera, S.P.G., A.T. Parra, L.F.C. Zapata \& M.Z. Lopez, 2004. Efeito inseticida y residual de três extractos de Lippia Alba para el control de Acanthocelides obtectus em frijol diacol calima. Revista Científica Guilhermo de Ockham, 7: 187-199.

Silva, G.C., D.P. Gomes \& C.C. Santos, 2011. Sementes de FeijãoCaupi (Vigna unguiculata L. (Walp), tratadas com extrato de folhas de nim (Azadirachta indica A. Juss.) avaliação da determinação e da incidência e fungos. Scientia Agraria, 12: 019-024. DOI: https://doi.org/10.5380/rsa.v12i1.33669.

Silva, G.N., L.R.A. Faroni, A.H. Sousa \& R.S. Freitas, 2012. Bioactivity of Jatropha curcas L. to insect pests of stored products. Journal of Stored Products Research, 48: 111-113. DOI: https://doi.org/10.1016/j.jspr.2011.10.009.

Soares, B.V. \& M. Tavares-Dias, 2013. Espécies de Lippia (Verbenaceae), seu potencial bioativo e importância na medicina veterinária e aqüicultura. Biota Amazônica, 3: 109-123. DOI: https://doi.org/10.18561/2179-5746/ biotaamazonia.v3n1p109-123.

\section{Suggestion citation:}

Santos, V.S., P.H. Silva \& L.E. Pádua, 2018. Bioatividade do óleo essencial de Lippia sidoides Cham. (alecrim-pimenta) sobre Callosobruchus maculatus (Fabr.) (Coleoptera: Crysomelidae). EntomoBrasilis, 11 (2): 113-117.

Available on: doi:10.12741/ebrasilis.v11i2.737
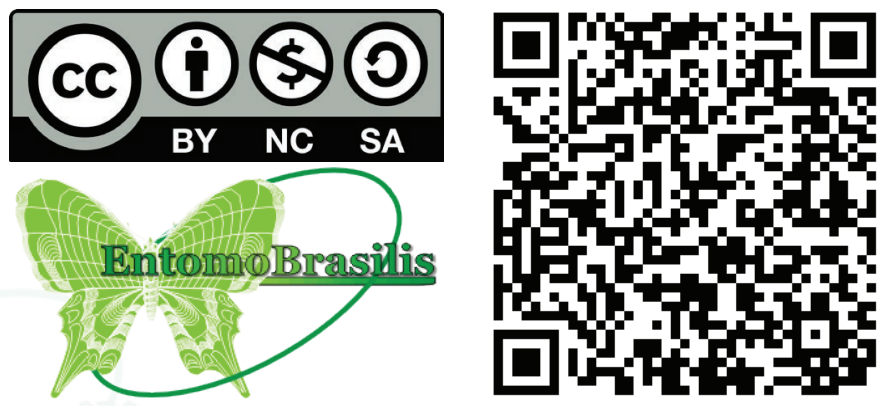